On the high-temperature behaviour of magnetically ordered microcrystals

This content has been downloaded from IOPscience. Please scroll down to see the full text.

1978 J. Phys. C: Solid State Phys. 11 L695

(http://iopscience.iop.org/0022-3719/11/16/004)

View the table of contents for this issue, or go to the journal homepage for more

Download details:

IP Address: 150.163.34.14

This content was downloaded on 25/08/2015 at 20:32

Please note that terms and conditions apply. 


\title{
On the high-temperature behaviour of magnetically ordered microcrystals
}

\author{
K Skeff Neto†, I C Cunha Limał, N S Almeida $\S$ and L C M Miranda§ \\ $\uparrow$ Departamento de Física, Universidade de Brasília, 70.000 Brasília, DF, Brazil \\ ¥Departamento de Engenharia Espacial, Instituto Nacional de Pesquisas Espaciais, 12200 \\ São José dos Campos, SP, Brazil \\ §Instituto de Física, Universidade Estadual de Campinas, 13100 Campinas, SP, Brazil
}

Received 18 May 1978

\begin{abstract}
A simple molecular-field model taking into account the characteristic volume distribution of an assembly of magnetically ordered microcrystals is developed to explain the observed high-temperature behaviour of the magnetisation. It is also shown that the volume distribution implies a distribution of critical temperatures.
\end{abstract}

The magnetic properties of microcrystals (systems consisting of an assembly of fine magnetic particles of 20-200 $\AA$ diameter) are currently a subject of considerable interest. A number of investigations on superparamagnetism (Wohlfarth 1977, Morup and Topsoe 1976) and the discovery of new phenomena like the surface sensitive magnetic anisotropy energy barriers (Dumeste et al 1975) and pinning of surface spins (Berkowitz et al 1975 ) have recently been reported. The original description of the magnetisation properties of such systems was suggested by Néel (1949): in these single domain particles the magnetisation vector is held in an easy direction by the crystalline field. At any finite temperature, thermal fluctuations cause the magnetisation to undergo a sort of Brownian rotation around the easy axis, and there is a finite probability that it will instantaneously change its direction into another easy direction. The relaxation time, which indicates how rapidly this transition occurs, was deduced by Néel and is given by Wohlfarth (1975), Morup and Topsoe (1976) and Néel (1949)

$$
\tau=\tau_{0} \exp \left(K V / k_{\mathrm{B}} T\right)
$$

where $\tau_{0}$ is a time of the order of $1 \mathrm{~ns}, K$ is the anisotropy constant, $V$ the volume of the microcrystal, and $k_{\mathrm{B}} T$ is the thermal energy.

In this Letter we report on a model calculation for the high-temperature behaviour of the magnetisation of magnetically ordered microcrystals, having a characteristic grain size distribution, and show that the observed one-third power law is adequately accounted for. We begin our discussion by noticing that in a measurement of the magnetic properties of a single-domain particle, what is observed depends on the ratio of the time required for the measurement, $\tau_{\mathrm{obs}}$, to the relaxation time, $\tau$, given by equation (1). For $\tau_{\text {obs }} \ll \tau$, the particles will show ferromagnetic behaviour whereas for $\tau_{\text {obs }} \gg \tau$, the 
particles will be superparamagnetic. With the Mössbauer spectroscopy, for instance, the nuclear Zeeman splitting caused by the internal magnetic field at the site of the nucleus can be observed only if the Larmor frequency $\nu_{\mathrm{L}}$ of the nuclear spin is larger than $\tau^{-1}$ (i.e., $\tau_{\text {obs }}$ corresponds to $v_{L}^{-1}$ ). For $\tau \gg v_{L}^{-1}$ the particles will be superparamagnetic and the Zeeman splitting will disappear; for $\tau \approx v_{L}^{-1}$ one observes a partially relaxed magnetic hyperfine spectrum. In this sense the Mössbauer spectroscopy is a particularly suitable tool for investigating superparamagnetism. As an example, in figure 1 we show

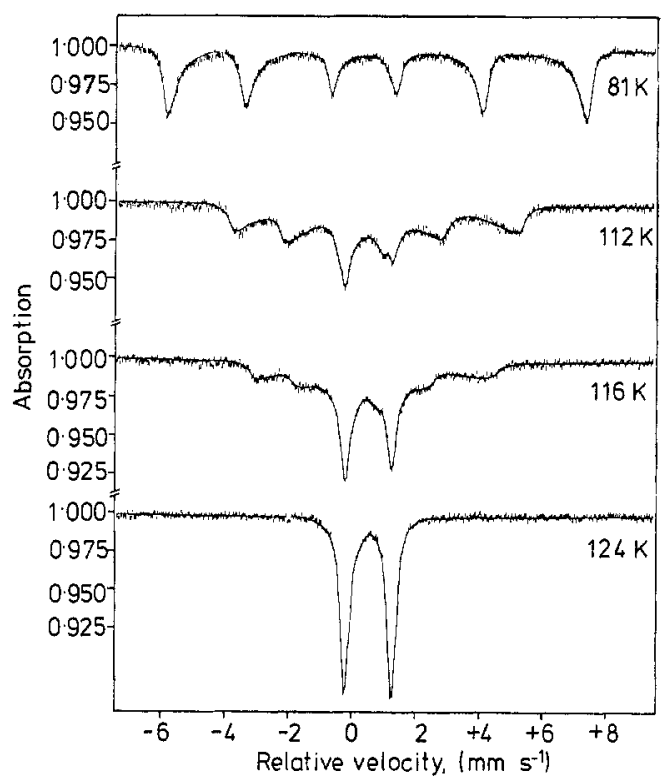

Figure 1. Mössbauer spectra of $\mathrm{FeOHSO}_{4}$ at different temperatures, taken from Skeff Neto and Garg (1975) and Skeff Neto and Miranda (1978).

the Mössbauer spectrum of $\mathrm{FeOHSO}_{4}$ at various temperatures. The experimental details as well as the sample preparation were reported elsewhere (Skeff Neto and Garg 1975, Skeff Neto and Miranda 1978). The Mössbauer spectrum at $T=112 \mathrm{~K}$ already shows a well-resolved six-peak pattern with a considerable broadening of the outer assymetric peaks and an enhancement of the electric quadrupole splitting lines. These features clearly suggest that there is a distribution of hyperfine fields reflecting all possible varieties of local environments (Tsuei and Lilienthal 1976, Sharon and Tsuei 1972) (i.e. the contribution of all microcrystals in the sample). In the following we show that this distribution of hyperfine fields is essentially due to a particle size distribution. Furthermore, this size distribution determines the observed temperature dependence of the magnetisation when one includes, in the description of our system, the particle anisotropy contribution, as in the usual superparamagnetic relaxation theories (Morup and Topsoe 1976, Néel 1949). To this end we assume an assembly of magnetically ordered particles having a size distribution $P(V)$. The magnetic Hamiltonian for a particle of volume, $V$ with uniaxial symmetry may then be written as (Wohlfarth 1977, Morup and Topsoé 1976, Néel 1949) 


$$
\mathscr{H}=-J \sum_{i, j} S_{i} . S_{j}-K V \sum_{i}\left(S_{i}^{z}\right)^{2}
$$

where $J$ is the Heisenberg exchange integral between the $z$ nearest-neighbour spins $(S)$, and $K V$ is the anisotropy energy barrier. Within the molecular-field approximation and for $S=\frac{1}{2}$, it follows from equation (2) that the magnetisation per atom $M=\left\langle S_{i}^{z}\right\rangle$ (i.e., the thermodynamic average of the $z$-component of the spin) can be easily calculated and the result is

$$
M(V)=\frac{1}{2} \tanh [\beta(J z+K V) M(V) / 2]
$$

where $\beta=1 / k_{\mathrm{B}} T$. Expanding equation (3) in the limit when $M(V) \rightarrow 0$ the transition temperature $T_{\mathrm{c}}(V)$ is readily obtained. One gets

$$
k_{\mathrm{B}} T_{\mathrm{c}}(V)=(\mathrm{J} z+K V) / 4 .
$$

The above equation establishes the transition temperatures for a grain of volume $V$. Since one has a distribution of volume, equation (3) implies that we also have a distribution of critical temperatures.

Now, if one realises that what, for instance, the Mössbauer spectrum gives us is the sum of contributions of the magnetisation of all microcrystals, the experimentally observed value $M$ of the sample magnetisation is then given by the averaged value of $M(V)$ over the volume distribution, namely,

$$
M=\int_{0}^{\infty} \mathrm{d} V P(V) M(V) .
$$

Here $M(V)$ is given by equation (3) and $P(V)$ is the volume distribution which may be known from, say, electron microscopy data. Equation (5) established the connection between our model and the experiment. To proceed we shall further assume a Lorentzian distribution of volume with average volume $V_{0}$ and width $\Gamma$, i.e.,

$$
P(V)=\frac{\Gamma / 2}{(\pi / 2)+\tan ^{-1}\left(2 V_{0} / \Gamma\right)} \frac{1}{\left(V-V_{0}\right)^{2}+(\Gamma / 2)^{2}} .
$$

This representation for $P(V)$ is generally accepted. In particular, in the case of $\mathrm{FeOHSO}_{4}$,

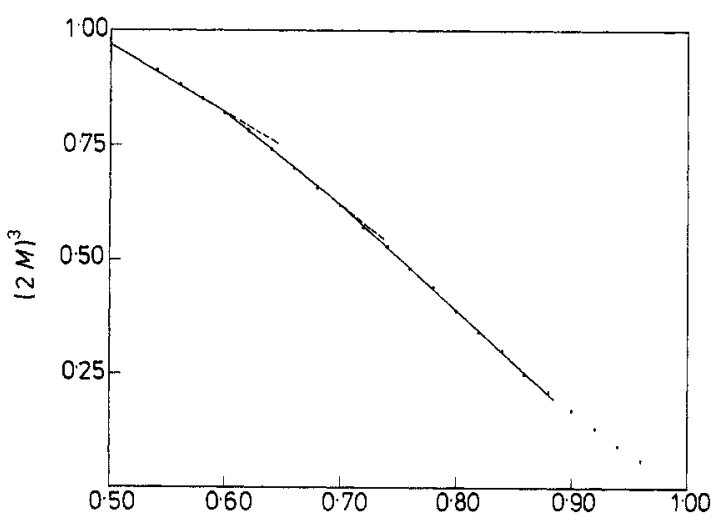

Figure 2. Varition of the magnetisation cubic (compare equations (3), (5) and (6)) versus the reduced temperature for $\alpha=1.01$ and $\eta=0.30$. 
Table 1.

\begin{tabular}{|c|c|c|c|c|}
\hline $\begin{array}{l}(2 M)^{3} \\
\tau\end{array}$ & $\begin{array}{l}\alpha=0.51 \\
\eta=0.20\end{array}$ & $\begin{array}{l}0.51 \\
0.30\end{array}$ & $\begin{array}{l}1 \cdot 01 \\
0 \cdot 20\end{array}$ & $\begin{array}{l}1.01 \\
0.30\end{array}$ \\
\hline 0.50 & 0.92 & 0.98 & 0.91 & 0.97 \\
\hline 0.52 & 0.90 & 0.96 & 0.89 & 0.94 \\
\hline 0.54 & 0.87 & 0.93 & 0.86 & 0.91 \\
\hline 0.56 & 0.84 & 0.90 & 0.83 & 0.88 \\
\hline 0.58 & 0.81 & 0.87 & 0.80 & 0.85 \\
\hline 0.60 & 0.78 & 0.83 & 0.76 & 0.82 \\
\hline $0 \cdot 62$ & 0.74 & 0.79 & 0.73 & $0 \cdot 78$ \\
\hline 0.64 & 0.70 & 0.76 & 0.69 & 0.74 \\
\hline 0.66 & 0.66 & 0.72 & 0.65 & 0.70 \\
\hline 0.68 & 0.62 & 0.67 & 0.61 & 0.66 \\
\hline 0.70 & 0.58 & 0.63 & 0.57 & $0 \cdot 62$ \\
\hline 0.72 & 0.54 & 0.58 & 0.53 & 0.57 \\
\hline 0.74 & 0.49 & 0.54 & 0.48 & 0.53 \\
\hline 0.76 & 0.45 & 0.49 & 0.44 & 0.48 \\
\hline 0.78 & 0.40 & 0.44 & 0.40 & 0.44 \\
\hline 0.80 & 0.36 & 0.40 & 0.35 & 0.39 \\
\hline 0.82 & 0.31 & 0.35 & 0.31 & 0.34 \\
\hline 0.84 & 0.26 & 0.30 & 0.26 & 0.30 \\
\hline 0.86 & 0.22 & 0.25 & 0.22 & 0.25 \\
\hline 0.88 & 0.17 & 0.21 & 0.17 & 0.21 \\
\hline 0.90 & $0 \cdot 13$ & 0.17 & 0.13 & 0.17 \\
\hline 0.92 & 0.09 & 0.12 & 0.10 & 0.13 \\
\hline 0.94 & $0-06$ & 0.09 & 0.06 & 0.09 \\
\hline 0.96 & $0 \cdot 03$ & 0.05 & 0.03 & 0.06 \\
\hline 0.98 & 0.01 & 0.02 & 0.01 & 0.03 \\
\hline 1.00 & 0.00 & 0.01 & 0.00 & 0.01 \\
\hline
\end{tabular}

$V_{0}$ and $\Gamma$ are typically of the order of $0.52 \times 10^{-18} \mathrm{~cm}^{3}(d=100 \AA)$ and $4.19 \times 10^{-21}$ $\mathrm{cm}^{3}(\Delta=20 \AA)$, respectively (C A Ribeiro, unpublished).

In figure 2 we show the behaviour of $(2 M)^{3}$ as a function of the reduced temperature $\tau=T /\left\langle T_{\mathrm{c}}\right\rangle$ (where $k_{\mathrm{B}}\left\langle T_{\mathrm{c}}\right\rangle=\left(J z+k V_{0}\right) / 4$ ) for $\alpha=k V_{0} / J z=1 \cdot 01$ and $\eta=\Gamma / V_{0}=$ 0.30 . The computer data is summarised in table I. It follows from figure 2 and the analysis of table 1 that, in the high-temperature region, the $(2 M)^{3}$-curve is well represented by three successive straight lines indicating that $M$ obeys a one-third power law. The successive bendings are attributed to the fact that we have a volume distribution and that the larger particles take longer (according to equation (1)) to get disordered Finally, one notices that in the temperature region around $0.90,(2 M)^{3}$ is no longer a straight line. This is due to the fact that actually $P(V)$ is not truly symmetric. The region of high $V$ vanishes more rapidly than the low $V$ region. This is a consequence of the upper critical size, first predicted by Frenkel and Dorfman (1930), below which a particle of ferromagnetic material would consist of a single domain and be in a state of uniform magnetisation. This critical particle size was later estimated by Kittel (1946) and an approximate figure for the radius of a spherical sample of common ferromagnetic materials was found to be $150 \AA$. The kind of behaviour depicted in figure 2 is actually what is observed via Mössbauer spectroscopy in several systems of magnetically ordered microcrystals (Skeff Neto and Garg 1975, Skeff Neto and Miranda 1978, Kundig et al 1966). As an example 


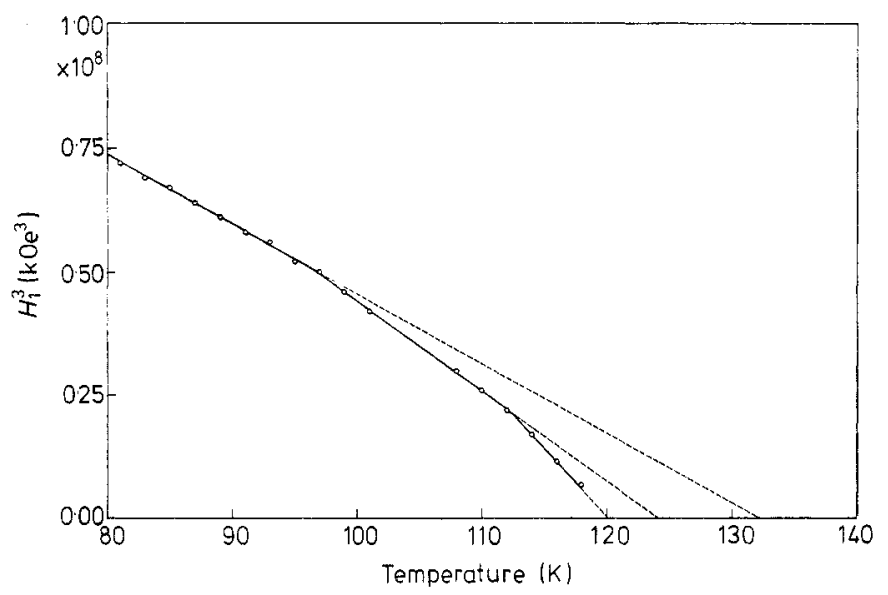

Figure 3. Variation of the internal field cubic with temperature for $\mathrm{FeOHSO}_{4}$ taken from Skeff Neto and Miranda (1978). The experimental points are represented by circles.

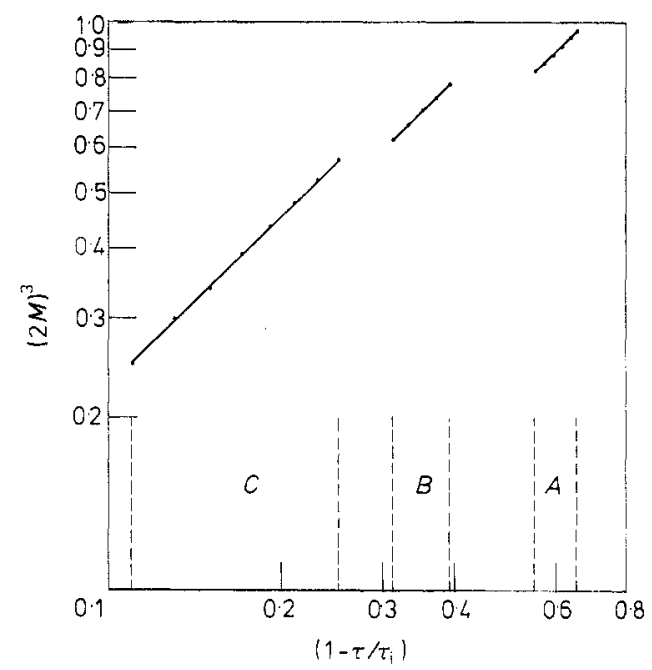

Figure 4. Variation of $(2 M)^{3}$ as a function of $\left(1-\tau / \tau_{i}\right)$ in a double-log scale for $\alpha=1.01$ and $\eta=0 \cdot 30$. The symbols are the same as those defined in the text.

we show in figure 3 the plot of the internal field cubic, $H_{i}^{3}$, versus the temperature for $\mathrm{FeOHSO}_{4}$ (Skeff Neto and Garg 1975, Skeff Neto and Miranda 1978). Finally, to best depict the one-third power law we show in figure 4 the $\log$-log plot of $(2 M)^{3}$ as a function of $\left(1-\tau / \tau_{i}\right)$. Here $\tau_{i}(i=A, B$, or $C)$ refers to the critical temperature in regions $A$ $(0.50 \leqslant \tau \leqslant 0.60), B(0.62 \leqslant \tau \leqslant 0.70)$ and $C(0.72 \leqslant \tau \leqslant 0.86)$, and are obtained by the extrapolation of the three successive straight lines of figure 2 ; we have $\tau_{\mathrm{A}}=1.15$, $\tau_{B}=1.01$ and $\tau_{C}=0.97$. The angular coefficient one gets in these regions are $\tau_{A}=1.00$, $\tau_{\mathrm{B}}=1.00$ and $\tau_{\mathrm{C}}=0.99$ respectively.

In conclusion, we have demonstrated in this Letter that within the molecular-field 
approximation, the volume distribution of a system of magnetically ordered particles is largely responsible for the magnetic properties of these systems. In particular it is responsible for the distribution of critical temperature characteristic of the systems as well as for the observed one-third power law for the magnetisation as a function of the temperature.

\section{References}

Berkowitz A E, Lahut J A, Jacobs I S, Levison L M and Forester D W 1975 Phys. Rev. Lett. 34594

Dumeste J A, Topsoe H and Boudart M 1975 J. Catal. 37113

Frenkel J and Dorfman J 1930 Nature 126274

Jacobz I S and Bean C P 1963 in Magnetism ed G T Rado and M Sohl, vol III (New York: Academic) p 271

Kittel C 1946 Phys. Rev. 70965

Kundig W, Bummel H, Constabaris G and Lindquist R H 1966 Phys. Rev. 142327

Morup S and Topsoe H 1976 Appl. Phys. 1163

Néel L 1949 Ann. Geophys. 599

Roggwiller P and Kundig W 1973 Solid St. Commun. 12901

Sharon T E and Tsuei C C 1972 Phys. Rev. B 51047

Skeff Neto K and Garg V K 1975 J. Inorg. Nucl. Chem. 372287

Skeff Neto K and Miranda L C M 1978 Phys. Rev. B (submitted for publication).

Tsuei C C and Lilienthal H 1976 Phys. Rev. B 134899

Wohlfarth E P 1977 Physica 86-88 B 852 